\title{
Comparisons of Faulting-Based Pavement Performance Prediction Models
}

\author{
Weina Wang, ${ }^{1}$ Yu Qin, ${ }^{2}$ Xiaofei Li, ${ }^{3}$ Di Wang, ${ }^{4}$ and Huiqiang Chen ${ }^{3}$ \\ ${ }^{1}$ State and Local Engineering Laboratory for Civil Engineering Material, School of Civil Engineering, Chongqing Jiaotong University, \\ Xuefu Avenue No. 66, Nanan District, Chongqing, China \\ ${ }^{2}$ CREEC (Chongqing) Survey, Design and Research Co. Ltd., Kunlun Avenue No. 46, Liangjiang New Area, Chongqing, China \\ ${ }^{3}$ School of Civil Engineering, Chongqing Jiaotong University, Xuefu Avenue No. 66, Nan'an District, Chongqing, China \\ ${ }^{4}$ Pavement Engineering Centre, Technical University of Braunschweig, Raum 104, Beethovenstraße 51 b, Braunschweig, Germany
}

Correspondence should be addressed to Yu Qin; qinyubridge@163.com

Received 15 April 2017; Revised 2 July 2017; Accepted 6 August 2017; Published 18 September 2017

Academic Editor: Hainian Wang

Copyright (C) 2017 Weina Wang et al. This is an open access article distributed under the Creative Commons Attribution License, which permits unrestricted use, distribution, and reproduction in any medium, provided the original work is properly cited.

\begin{abstract}
Faulting prediction is the core of concrete pavement maintenance and design. Highway agencies are always faced with the problem of lower accuracy for the prediction which causes costly maintenance. Although many researchers have developed some performance prediction models, the accuracy of prediction has remained a challenge. This paper reviews performance prediction models and JPCP faulting models that have been used in past research. Then three models including multivariate nonlinear regression (MNLR) model, artificial neural network (ANN) model, and Markov Chain (MC) model are tested and compared using a set of actual pavement survey data taken on interstate highway with varying design features, traffic, and climate data. It is found that MNLR model needs further recalibration, while the ANN model needs more data for training the network. MC model seems a good tool for pavement performance prediction when the data is limited, but it is based on visual inspections and not explicitly related to quantitative physical parameters. This paper then suggests that the further direction for developing the performance prediction model is incorporating the advantages and disadvantages of different models to obtain better accuracy.
\end{abstract}

\section{Introduction}

Transverse joint faulting is the common type of distress for jointed concrete pavement, which has negative effect on driving safety and resulting costly rehabilitation [1]. Pavement faulting prediction is essential for concrete pavement management system and pavement design strategy. And pavement maintenance decision-making is based on current and future conditions. In the past decades, many researchers have been focusing on the developing of pavement performance prediction and improving its accuracy. However, the changing of pavement performance is a complex processing; establishing an easy and accurate predicted model has remained a challenge. Therefore, it is necessary to study the performance of various pavement prediction models.

The paper first presents a comprehensive literature review that discusses previous work on the pavement performance prediction and its classification. Three different models including MNLR model, ANN model, and MC model are briefly introduced. These models are quantitatively evaluated and compared using a set of concrete pavement survey faulting data with varying design features, traffic, and climate data. These survey faulting data are taken for evaluating the performance of the three models. The results of these prediction models are presented. Then the strengths and weaknesses of these models are surveyed. Finally, the areas of concern in performance prediction and potential for future work are addressed. Suggestions for future research work are proposed by incorporating the advantages and disadvantages of different models.

\section{Literature Survey}

According to the prediction results, the performance models can be divided into deterministic and probabilistic models. For the deterministic models, future condition of pavement 
section was predicted as the exact serviceability value or pavement condition index with the previous information of the pavement. The probabilistic models predict the performance of a pavement by giving the probability with which the pavement would fall into a particular condition state, describing the possible pavement conditions of the random process [2]. Deterministic models included mechanistic model, empirical model, and mechanistic-empirical models. Probabilistic models included Markov models, Bayesian approach, and survival analysis.

2.1. Deterministic Model. Deterministic models are perhaps the most common prediction method. The main advantages of using a deterministic model are easy to understand and develop. Disadvantage of deterministic models is that the regression equation may express the deterioration of a group of pavements well but do not predict the condition of individual sections very well [3]. Deterministic models can usually be categorized as mechanistic, empirical, and mechanistic-empirical models.

Mechanistic models are based on the principles of mechanics of materials and use the input of wheel loads to predict the mechanistic responses, such as stress, strain, and deflection. The mechanistic models provide valuable insights into the performance of the pavements. There are some researchers focused on mechanistic model. Chua et al. established the distress model that defined a performance function in which the level of distress may be determined as a function of the controlling structural response according to some damage criterion [4]. The continuous reinforced concrete pavement computer program by National Cooperative Highway Research Program (NCHRP) has been modified several times to expand the ability of the mechanistic model [5]. Al-Qadi et al. also establish mechanistic pavement model by finite element method and then in situ validation is also taken [6]. Since the deterioration process of pavement performance is complex and not completely understood, pure mechanistic models developed so far cannot accurately predict the realistic pavement performance [7]. Therefore, the development of reliable and acceptable mechanistic models requires a significant amount of time and effort for continuous studies.

Empirical approach is widely used in the prediction area, but it suffers from the limitations associated with the scope and range of available data. As an empirical model, the most important pavement performance model is created by American Association of State Highways Officials, and it is based on the results of actual road test [8]. In a SHRP study conducted by Simpson et al., based on early analysis of Long-Term Pavement Performance (LTPP) general pavement studies data, JPCP faulting models are developed, which are empirical models [9]. Prozzi and Madanat use joint estimation techniques to combine experimental data and field data for modeling the pavement performance [10].

There are also some JPCP transverse joint faulting empirical models developed under previous research. Yu develops two separate JPCP faulting models for doweled and nondoweled pavements as part of FHWA RPPR project. The development of these models identifies several pavement design features and site conditions that significantly affect transverse joint faulting. Teng develops separate mechanisticempirical JPCP faulting models for doweled and nondoweled pavements for American Concrete Paving Association (ACPA) [11].

Mechanistic-empirical models are those in which responses predicted by mechanistic models were correlated with usage or environmental variable such as loadings or age to predict observed performance, such as distress. Most mechanistic-empirical models are used for the project level. Few were used for the network level. However, as the speed and capacity of microcomputers increase and the cost of collecting more structural information decreases, these mechanistic-empirical models are used more and more in prediction models [12]. Under the support of Federal Highway Administration (FHWA), Teng establishes the mechanistic-empirical distress indicator prediction models and develops software Pave Spec 3.0 for JPCP [11]. The Mechanistic-Empirical Pavement Design Guide (MEPDG, 2004) is also developed to replace the AASHTO 1993 Guide for the Design of Pavement Structure [13]. Most of distress prediction models in design guide are empirical-mechanistic models, which causes wide public concern.

In addition, especially for faulting model, there are also some research works on this. Under the FHWA Nationwide Pavement Cost Model (NAPCOM) study, Owusu-Antwi develops the following mechanistic-empirical faulting model for doweled and nondoweled JPCP. Titus Glove recalibrates NAPCOM JPCP transverse joint faulting model by LTPP data. But this model is recalibrated using LTPP data only [11]. Ker et al. also establish mechanistic-empirical faulting prediction models for rigid pavements using LTPP database [14]. Jung and Zollinger present a mechanistic-empirical faulting model, which is calibrated from the results of a new erosion test that involves the Hamburg wheel-tracking device and LTPP data [15].

2.2. Probabilistic Model. Pavement performance is a stochastic process that varies widely with several factors, many of which are generally not captured by available data. Therefore, probabilistic models are often used to characterize performance. The following list summarizes the major advantages and disadvantages associated with probabilistic model [16].

The major advantages associated with probabilistic modeling approaches are as follows:

(1) They provide a convenient way to incorporate field data into a prediction model.

(2) They leave it to subjective inputs of experienced agency personnel.

(3) They provide a mathematical means for obtaining performance predictions.

(4) They provide a probabilistic distribution of the expected condition value with time, which will be required to identify those sections performing significantly differently than would be expected.

(5) They reflect performance trends obtained from field observations regardless of nonlinear trends with time. 
The major disadvantages associated with probabilistic models are listed:

(1) They do not provide any guidance as to the physical factors that contribute to the change in condition.

(2) They are time independent so that the probability of changing from one condition state to a lower condition state is not influenced by the age of the pavement and the probabilities are constant over time.

Probabilistic models include Markov models, survival analysis, and Bayesian approach.

Markov Chains based on the concept of probabilistic cumulative damage are the most commonly used stochastic techniques for predicting the performance of various infrastructure facilities such as highways and bridges. Lounis and Madanat combine the desired practicality of Markov Chain models and the accuracy of mechanistic models to improve the effectiveness of bridge maintenance management systems [17]. Golroo and Tighe apply a combination of homogeneous and nonhomogeneous Markov Chain to develop performance model [18]. Pulugurta et al. developed a Markov prediction model using the pavement condition database of Ohio Department of Transportation [19]. Lethanh and Adey established exponential hidden Markov models for roughness and texture depth indices [20]. Abaza used simplified staged-homogenous Markov model for flexible pavement performance prediction at the project level [21].

Survival analysis is generally defined as a set of methods for analyzing data where the outcome variable is the time until the occurrence of an event of interest, which has been used in the performance prediction. Survival methods include parametric, nonparametric, and semiparametric approaches. Parametric methods assume that the underlying distribution of the survival times follows certain known probability distributions. Weibull model is the popular one. Mishalani et al. develop a probabilistic model with Weibull distribution function in different areas [22-24]. Cox model, known as the proportional hazard model, is one of the most popular models in the semiparametric models [25]. Mauch and Madanat use the Cox proportional hazards model to create a more descriptive model of deterioration without prespecifying distributions for parameters relating independent variables and deterioration [26]. Nakat and Madanat also use a semiparametric Cox model to develop a pavement cracking model [27]. As a nonparametric estimator of the survival function, the Kaplan-Meier method is widely used to estimate and graph survival probabilities as a function of time. Chou et al. used Kaplan-Meier method to estimate the median survival time to the next treatment for pavement performance [28]. Based on Kaplan-Meier method, Pulugurta also develops survival curves using available historical pavement data [29].

Bayesian approach can be used with most approaches, except the truly mechanistic models [10]. Hong and Prozzi develop the pavement deterioration forecasting model based on the Bayesian approach and Markov model and use Bayesian approach to obtain probabilistic parameter distributions through a combination of existing knowledge priorly and information from the data collected [30]. Morcous develops a performance prediction of bridge deck systems using Markov Chains and Bayesian approach [31]. Gao et al. propose modeling the fatigue cracking of flexible pavement by means of survival model and adopt Bayesian approach by using a Markov Chain Monte Carlo (MCMC) algorithm [32]. It is shown that various modifications to each of these types of models can be used and it is in development.

2.3. Other Models. ANN models are varied in implementation and interpretation. An ANN is a mathematical representation of how mammalian brains were believed to function [8]. Essentially an ANN model functions like a regression equation, in that a number of parameters variables are used to predict a dependent variable from a number of independent ones. However, unlike a regression equation that depended upon the ability of designer to comprehend the form of equation a priori, neural networks use their internal massively parallel structure to determine relationships with no input from the designer. Thus, a neural network is a tool which was used in most of the performance prediction area.

The advantage of artificial neural networks is their ability to be trained on previous situations. Training is required to continuously adjust the connection weights until they reach values that allowed ANN to predict outputs that are very close to the actual outputs while being able to be generalized well on new cases [33].

Some of the disadvantages for ANN are as follows [8]:

(1) Prohibitively slow training times for large networks

(2) Problems with previously unrepresented patterns in supervised training

(3) Ideal network architectures and training algorithms remaining part of current research

(4) Problems with local minima in training

(5) Lack of ability to explain mechanisms in predictive models.

ANN can be used in the performance prediction in different areas. Tack and Felker used the ANN method to predict the performance and it is provided that this method performs well $[8,34]$. Huang develops an application model based on ANN approach for estimating the future condition of bridges [35]. Karwa and Donnell use ANN to predict pavement marking retroreflectivity by data from North Carolina [36]. Saghafi et al. use ANN approach for predicting faulting considering base condition, and it is indicated that ANN approach can predict joint faulting in jointed concrete pavements successfully [37]. Recently, ANN models are commonly used in pavement performance models $[38,39]$.

2.4. Summary. The overview of existing literature reflects some prominent problems in the prediction area. First, there are many types of prediction models. The advantages and disadvantages for each type are also introduced. But, based on the advantages and disadvantages, it is hard to estimate and compare the predicted performance for each model. 
Many models are able to perform well only in a dataset but not in different dataset. Hence, calibrations are required to adjust the parameter inputs so that the models can perform reasonably. Second, not all the prediction models can be used in the special case, for they may lack some parameters that cannot be acquired or there are not enough actual data to establish the model we are choosing. So evaluating the effectiveness of existing models on the same actual dataset that had variable design features, traffic, and climate is essentially useful for researchers.

For JPCP faulting models, existing researches identified a number of distinct relationships between faulting and traffic, age, and various climatic, site, and pavement design variables. All of the models indicate that design features have a significant effect on faulting. These models are almost either empirical models or mechanistic-empirical models, which also suffers from the disadvantages of these types of models. But the review of these developed transverse joint faulting models identified a number of variables that have been consistently found to significantly influence faulting.

Hence, in this paper we make a quantitative comparison of three different prediction models with actual survey data being conducted. Two are the JPCP faulting prediction models, while the other two are the prediction methods that can be used in this faulting prediction. It is hoped that the comparison results will provide crucial information for researchers and state DOTs on developing enhanced pavement performance models that can lead to a more accurate prediction for maintenance system and design system.

\section{Data Preparation}

Actual pavement survey data used in the models are taken from interstate highway with varying design features, traffic, and climate data ([40], Web-1: http://www.noaa.gov/). There are 9 sections with 143 records for this whole dataset. These 143 datasets are divided into two parts: 107 records (approximately $75 \%$ of the whole dataset) are used for training. 36 records (approximately $25 \%$ of the whole dataset) are used for prediction. These 36 records are the last 4 years' records for each section. Training set is different from those prediction sets and it is greater than prediction set. Faulting distribution is presented in Figure 1.

\section{Models Used for Comparison}

Based on the modeling methodology, it is found that different types of models have different characteristics. It is important to understand the feasibility of each prediction model by comparing their results. Since pavement performance deterioration process is complex and not completely understood, the pure mechanistic models developed so far cannot accurately predict the realistic pavement performance. Therefore, mechanistic model is not chosen in the paper.

MNLR model is a primal, useful technique which has been applied in all fields of engineering knowledge. ANN model, neither deterministic nor probabilistic, can be used in all the performance predictions and performs well [37], now being widely used. MC model as a probabilistic model is the

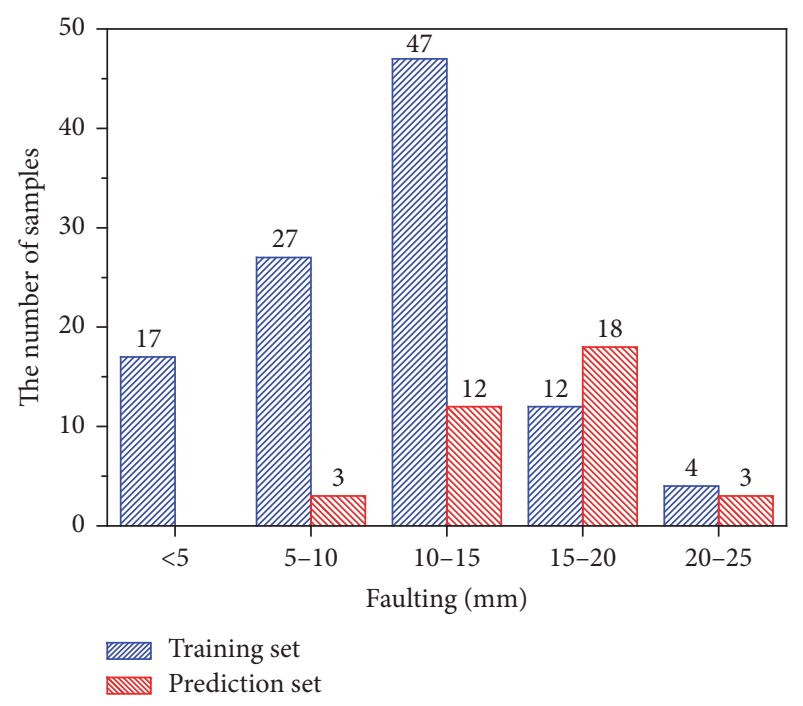

FIGURE 1: Faulting distribution of training set and prediction set.

most commonly used stochastic technique for predicting the various performances, which is practical and relatively easy to develop [31].

Based on the previous study, eight important factors that have greater impacts on faulting are used in the modeling [41]. They are ESAL, age, dowel diameter, base type, thickness, drainage, average annual rainfall, and freeze-thaw cycle times. The eight important factors that affected faulting are used in multivariate nonlinear regression (MNLR) model and artificial neural network (ANN) model. MC model is just associated with the faulting value and irrelevant to other important factors.

For the reasons listed above, MNLR model, ANN model, and MC model are used for comparative study. The modeling methods were described as follows.

4.1. MNLR Model. Equation (1) is the form of a multivariate nonlinear regression (MNLR) model to predict faulting, which performs well [42].

$$
\begin{gathered}
\text { FAULTING }=\mathrm{CESAL}^{a}[b+c \times \mathrm{AGE}+d \\
\times \text { DOWELDIA }+e \times \mathrm{BASE}+f \times \text { THICKNESS } \\
+g \times \text { DRAIN }+h \times \text { RAINFALL }+i \times \text { FTCYC }]
\end{gathered}
$$

where FAULTING is the faulting values, mm; CESAL are the accumulate equivalent single-axle loads; AGE is the pavement age; DOWELDIA is the dowel diameter, in; BASE is associated with erosion, defined as 1 to 5; THICKNESS is the slab thickness, in; DRAIN is the capability for drainage, defined as 0 to 1 ; RAINFALL is the average annual rainfall, $\mathrm{mm}$; FTCYC are the freeze-thaw cycle times; and $a, b, c, d, e$, $f, g, h, i$ are the regression coefficients.

4.2. ANN Model. Artificial neural network (ANN) is mathematical models and algorithms designed to mimic the information processing and knowledge acquisition that takes place inside human brain. ANNs are capable of learning 
by example. The back propagation neural network (BPNN) developed by Rumelhart et al. is the most representative learning model for the ANN. BPNN is widely applied in a variety of scientific areas, especially in applications involving diagnosis and prediction [37]. Back propagation is a systematic method that uses gradient descent based delta learning rule also known as back propagation rule for training multilayer feed forward artificial neural networks. The back propagation network design is a three-layer network with one of each input, hidden, and output layer. After evaluated computation, the best results were obtained by an 8-8-1 network structure. It has 8 neurons in the input layer, 8 neurons in the hidden layer, and 1 neuron in the output layer.

4.3. Markov Chain Model. Markov Chain (MC) model is a probabilistic model widely spread in the world. A Markov Chain is a special case of the Markov process whose development can be treated as a series of transitions between certain states. A stochastic process is considered as first-order. The probability of the future state in the Markov process depends only on the present state [31]. This property can be expressed for a discrete parameter stochastic process $\left(X_{t}\right)$ with a discrete state space as

$$
\begin{aligned}
& P\left(X_{t+1}=i_{i+1} \mid X_{t}=i_{t}, X_{t-1}=i_{t-1}, \ldots, X_{1}=i_{1}, X_{0}\right. \\
& \left.\quad=i_{0}\right)=P\left(X_{t+1}=i_{i+1} \mid X_{t}=i_{t}\right),
\end{aligned}
$$

where $i_{t}$ is state of the process at time $t$ and $P$ is conditional probability of any future event given the present and past events.

Transition probabilities are obtained from the increment of condition data to provide a better prediction [43]. Transition probabilities are represented by a matrix of order $(n \times n)$ called the transition probability matrix $(P)$, where $n$ is the number of possible condition states. Each element $\left(P_{i, j}\right)$ in this matrix represents the probability that the condition of a faulting increment component will change from state $(i)$ to state $(j)$ during a certain time interval called the transition period. If the initial condition vector $P(0)$ that describes the present condition of a faulting increment component is known, the future condition vector $P(t)$ at any number of transition periods $(t)$ can be obtained as follows:

$$
\begin{aligned}
P(t) & =P(0) \times P^{t} \\
P & =\left[\begin{array}{cccc}
P_{1,1} & P_{1,2} & \cdots & P_{1, n} \\
P_{2,1} & P_{2,2} & \cdots & P_{2, n} \\
\cdot & \cdot & \cdots & \cdot \\
P_{n, 1} & P_{n, 2} & \cdots & P_{n, n}
\end{array}\right] .
\end{aligned}
$$

When the predicted value is gotten, it is applied in next year's prediction. Then the new transition probability matrix with the predicted value is computed and next year's predicted value is obtained.

\section{Results}

Three prediction models are used to evaluate the capability of different models for predicting the pavement performance.
Here we present the results of three prediction models with 36 records. For comparing the capabilities of these proposed models, measured faulting and predicted faulting are both used. A summary of experimental results is presented in Figure 2.

Figure 2(a) represents the comparison of measured and predicted faulting by MNLR model. For this model, most predicted values are smaller than the measured values. The difference is nearly $5 \mathrm{~mm}$. The ability of predicting future value is not good. It may result in the range of initial data using for model regression. As an empirical model, the various data are really important for the predicted correction. Then more data is needed for recalibrating the MNLR model.

Figure 2(b) presents the relationship between measured faulting and predicted faulting computed by ANN model. It is observed that predicted values are greater than measured value. The difference is nearly $2 \mathrm{~mm}$. Kumar and Minocha point out that the number of weights required to be trained in the ANN model will be very large [44]. The training dataset of 107 records used in this paper may be inadequate. Therefore, more data are required for retraining ANN model. Increasing the number of training dataset is really good for getting better predictions in further studying. And only factors that are statistically significant to the dependent variables can be included in a prediction model. Testing the significance of those factors is also important.

Figure 2(c) shows a plot of the measured and predicted faulting using MC model. It is revealed that the predictions perform well, which are all near to the equality line. However, this model is just based on the condition data. For instance, the prediction is only affected by the data quality and is not related to the design feature or climate. More data attribute more accurate transition probability matrix. The model without design features which is supposed to be applied in pavement design is still difficult. Moreover, Markov model assumes that the future status is only determined by current status based on the transition matrix, which implies that previous status has no impact on future status. So the important factors are not considered in Markov model and the feasibility for prediction needs further study.

Root mean squared error (RMSE) and mean absolute error (MAE) are used to quantify the prediction accuracy [37]. The computation of root mean squared error and mean absolute error is described as follows:

$$
\begin{aligned}
\text { RMSE } & =\sqrt{\frac{1}{n} \sum_{i=1}^{n}\left(x_{0}-x_{p}\right)^{2}}, \\
\text { MAE } & =\frac{1}{n} \sum_{i=1}^{n}\left|x_{0}-x_{p}\right|,
\end{aligned}
$$

where $x_{0}$ is the measured value, $x_{p}$ is the predicted value, and $n$ is the total number of observations.

RMSE and MAE of predicted and measured values for the three prediction models are compared. As shown in Figure 3, the RSME of MNLR model, ANN model, and MC model is 3.86, 2.08, and 1.7, respectively. The prediction capability of MNLR model is worse than ANN model and 


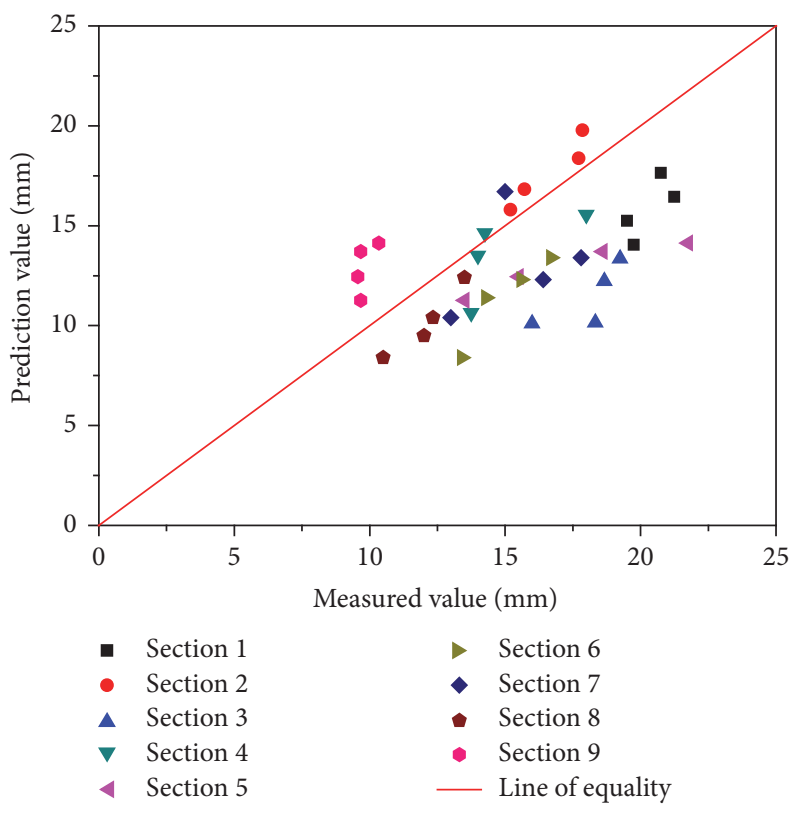

(a) MNRL model

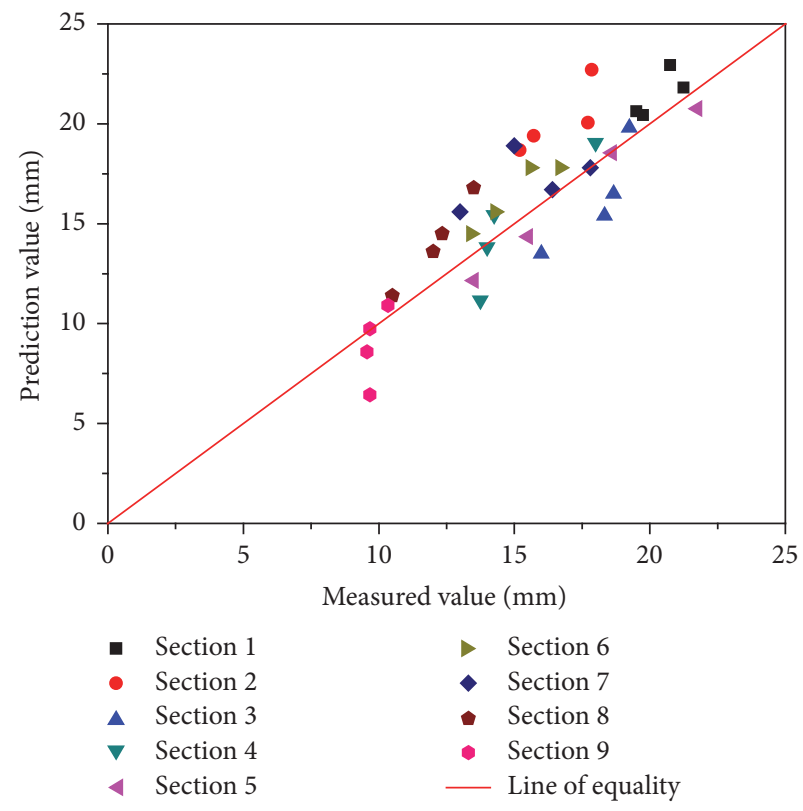

(b) ANN model

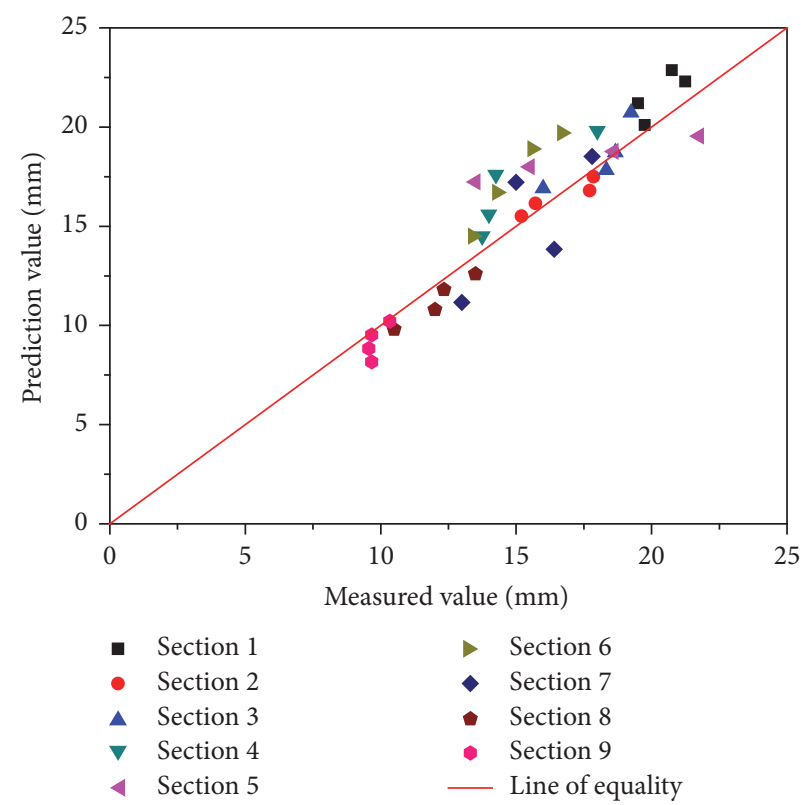

(c) MC model

FIGURE 2: Predicted faulting versus measured faulting.

MC model. ANN model and MC model perform well, and the difference for the prediction capability of these two is not obvious. The analysis of MAE that indicated a similar conclusion is presented. Previous study shows ANN model is more useful compared with World Bank developed model: the ANN model is applicable to all types of distress [45]. Also it is presented by other researchers that ANN also shows a higher capacity to predict joint faulting more accurately, compared with MLR (multivariate linear regression) model developed with the same data [37]. The prediction capacities of ANN model and regression model concluded in this paper are similar to others.

Figures 2 and 3 represent the best and worst model by using actual data. It is indicated that $\mathrm{MC}$ model can achieve a best predicted value, while MNLR model performs worst among the three. But for the limitation, MC model seems not to be the best model in the three. In summary, each model has its advantages and disadvantages; their own applicability is different. It is necessary to choose the right prediction model in the special case to get better performance. Based on the 


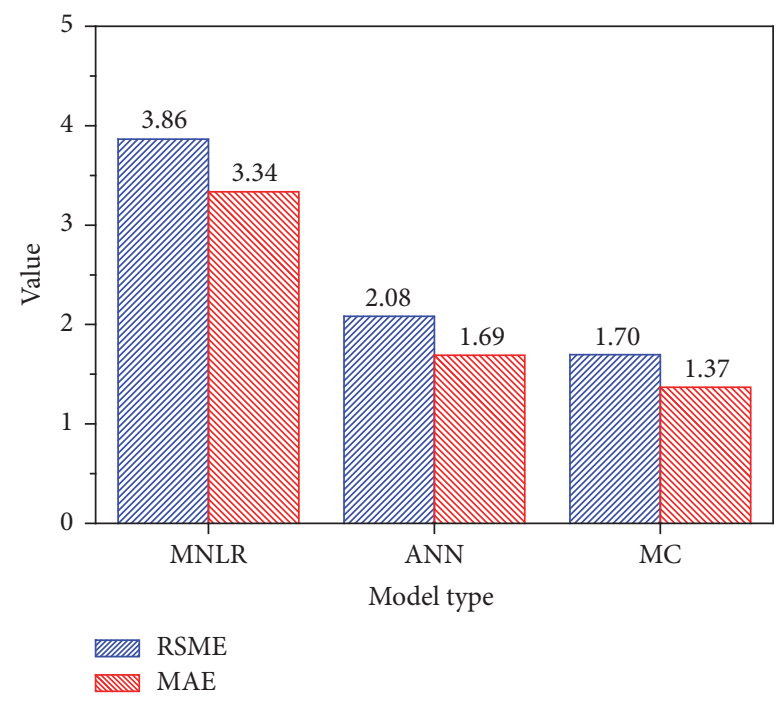

FIgURE 3: Quantitative comparison of different models.

results, there does not exist a no-disadvantage model in the representative model in each type of models. Developing a model having more advantages and fewer disadvantages is indeed essential in further study.

\section{Conclusions}

Although many researchers have developed pavement performance prediction models, the accuracy of the model is still a challenge. It is difficult to effectively compare the performance prediction models. Most researchers just focus on the optimization of the prediction model, but some researches do some work on the comparison of different models [37, 45, 46]. The advantages and disadvantages of each predicted model are introduced in many papers. But it is hard to know which model performs well just based on the summary of advantages and disadvantages for each model. Our research is motivated by this need to assess the performance of various prediction models. In this paper, we have conducted a comprehensive literature review of the various models used for performance prediction and JPCP faulting prediction. Three prediction models (MNLR model, ANN model, and MC model) are briefly introduced and their performance is quantitatively and objectively evaluated using the actual survey data.

Based on the test results, it is concluded that MNLR model performs the worst and MC model performs the best. ANN model and MC model perform well, and the difference of the prediction capabilities of these two is not obvious. MC model shows its promising performance compared with other models when data is limited. It is concluded in our comparative study that MC model is a promising model in prediction, but it is just based on its past condition and not related to the design feature and other environment factors. This characteristic makes it only applied in pavement maintenance, not in pavement design. ANN model performs better than MNLR model. MNLR model for its low predicted capacities needs more data to calibrate and ANN model also needs more data for training the network to improve its accuracy.

In the future, more prediction models can be tested using the actual survey data and compared with each other effectively. A bigger dataset that is composed of more complex situation is also needed in the model comparison. For different models having different effectiveness and applicability, it is important to find a developing and improving model to predict the pavement performance. Further direction for developing the performance prediction model is incorporating the advantages and disadvantages of different models to obtain better accuracy.

\section{Conflicts of Interest}

The authors declare that there are no conflicts of interest regarding the publication of this paper.

\section{Acknowledgments}

This paper is sponsored by National Natural Science Foundation of China [51508064, 51408083], China Postdoctoral Science Foundation [2014M562287], Chongqing Science and Technology Commission [cstc2014jcyjA30018, cstc2016jcyjA0128], Department of Human Resource and Social Security of Chongqing [Xm2014094], and State and Local Engineering Laboratory for Civil Engineering Material of Chongqing Jiaotong University [LHSYS-2016-01].

\section{References}

[1] J. S. Jung, Analytical Procedures for Evaluating Factors That Affect Portland Cement Concrete Pavement Performance [Ph.D. thesis], Arizona State University, Tempe, USA, 2004.

[2] W. D. Paterson, Road Deterioration and Maintenance Effects: Models for Planning and Management, John Hopkins University Press, Baltimore, USA, 1987.

[3] P. L. Durango, Adaptive Optimization Models for Infrastructure Management [Ph.D. thesis], University of California, Berkeley, USA, 2002

[4] K. H. Chua, C. L. Monismith, and K. C. Crandall, "Mechanistic Performance Model for Pavement Management," in Proceedings of the Third International Conference on Managing Pavements, Austin, USA, 1994.

[5] S. M. Kim, C. M. Won, and F. B. McCullough, "CRCP-9: Improved Computer Program for Mechanistic Analysis of Continuously Reinforced Concrete Pavements," Report No. 0-18312, Center for Transportation Research Bureau of Engineering Research. University of Teas at Austin, Austin, USA, 2001.

[6] I. L. Al-Qadi, M. A. Elseifi, and P. J. Yoo, "In-Situ Validation of Mechanistic Pavement Finite Element Modeling," in Proceedings of the 2nd International Conference on Accelerated Pavement Testing, Minneapolis, USA, 2004.

[7] Y. H. Huang, Pavement Analysis and Design, Pearson Prentice Hall, Pearson Education, Inc, Paramus, USA, 2nd edition, 2004.

[8] V. Felker, Characterizing the Roughness of KANSAS PCC and Superpave Pavements, [Ph.D. thesis], Kansas State University, Manhattan, 2005. 
[9] A. L. Simpson, J. B. Rauhut, P. R. Jordahl, E. Owusu-Antwi, I. M. Darter, and R. Ahmad, "Early analysis of LTPP general pavement studies data, volume III: sensitivity analyses for selected pavement distresses," Report No. SHRP-P-393, National Cooperative Highway Research Program, Transportation Research Board, Washington, DC, USA, 1994.

[10] J. A. Prozzi and S. M. Madanat, "Development of pavement performance models by combining experimental and field data," Journal of Infrastructure Systems, vol. 10, no. 1, pp. 9-22, 2004.

[11] T. P. Teng, "Improved Prediction Models for PCC Pavement Performance-Related Specifications," Volume: Final Report. Report No. FHWA-RD-00-130, National Cooperative Highway Research Program, Transportation Research Board, Washington, DC, USA, 2000.

[12] M. Nawaiseh, Pavement Performance Prediction Using Adaptive Logic Networks, [Ph.D. Thesis], Florida International University, Miami, Florida, FL, USA, 2002.

[13] ARA Inc, Guide for Mechanistic-Empirical Design of New and Rehabilitated Pavement Structures, Final Report - NCHRP Project 1-37A, National Cooperative Highway Research Program, Transportation Research Board, Washington, Wash, USA, 2004.

[14] H. W. Ker, Y. H. Lee, and C. H. Lin, "Development of faulting prediction models for rigid pavements using LTPP database," International Journal of Pavement Research and Technology, vol. 5, pp. 658-666, 2013.

[15] Y. Jung and D. Zollinger, "New laboratory-based mechanisticempirical model for faulting in jointed concrete pavement," Transportation Research Record, no. 2226, pp. 60-70, 2011.

[16] R. Haas, W. R. Hudson, and J. Zaniewski, Modem Pavement Management. Publication No. FHWA/OH-2004/016, Robert E. Krieger Publishing Company, Malabar, USA, 1994.

[17] Z. Lounis and S. M. Madanat, "Integrating Mechanistic and Statistical Deterioration Models for Effective Bridge Management," in Proceedings of the Seventh International Conference on Applications of Advanced Technologies in Transportation (AATT), pp. 513-520, Boston Marriot, Cambridge, Massachusetts, United States.

[18] A. Golroo and S. Tighe, "Use of soft computing applications to model pervious concrete pavement condition in cold climates," Journal of Transportation Engineering, vol. 135, no. 11, pp. 791800, 2009.

[19] H. Pulugurta, Q. Shao, and Y. J. Chou, "Pavement condition prediction using Markov process," Journal of Statistics and Management Systems, vol. 12, no. 5, pp. 853-871, 2009.

[20] N. Lethanh and B. T. Adey, "Use of exponential hidden Markov models for modelling pavement deterioration," International Journal of Pavement Engineering, vol. 14, no. 7, pp. 645-654, 2013.

[21] K. A. Abaza, "Simplified staged-homogenous Markov model for flexible pavement performance prediction," Road Materials and Pavement Design, vol. 17, no. 2, pp. 365-381, 2016.

[22] R. G. Mishalani and S. M. Madanat, "Computation of infrastructure transition probabilities using stochastic duration models," Journal of Infrastructure Systems, vol. 8, no. 4, pp. 139-148, 2002.

[23] C. Shin and S. Madanat, "Development of Stochastic Model of Pavement Distress Initiation, Applications of Advanced Technology in Transportation," 744, pp. 497-505, 2003.

[24] K. Kobayashi, K. Kaito, and N. Lethanh, "Deterioration forecasting model with multistage Weibull Hazard functions," Journal of Infrastructure Systems, vol. 16, no. 4, pp. 282-291, 2010.
[25] K. D. Kuhn, Uncertainty in Infrastructure Deterioration Modeling and Robust Maintenance Policies for [Ph.D. thesis], Markovian Management Systems, University of California, California, CA, USA, 2006.

[26] M. Mauch and S. Madanat, "Semiparametric hazard rate models of reinforced concrete bridge deck deterioration," Journal of Infrastructure Systems, vol. 7, no. 2, pp. 49-57, 2001.

[27] Z. S. Nakat and S. M. Madanat, "Stochastic duration modeling of pavement overlay crack initiation," Journal of Infrastructure Systems, vol. 14, no. 3, pp. 185-192, 2008.

[28] E. Chou, J. Tack, J. Yu, and J. Wielinski, "Evaluation of the Variation in Pavement Performance between ODOT Districts," Report No. FHWA/OH-2004/016, Federal Highway Administration, 2004.

[29] H. Pulugurta, Development of Pavement Condition Forecasting Models [Ph.D. thesis], University of Toledo, Toledo, Ohio, $\mathrm{OH}$, USA, 2007.

[30] F. Hong and J. A. Prozzi, "Estimation of pavement performance deterioration using Bayesian approach," Journal of Infrastructure Systems, vol. 12, no. 2, pp. 77-86, 2006.

[31] G. Morcous, "Performance prediction of bridge deck systems using markov chains," Journal of Performance of Constructed Facilities, vol. 20, no. 2, pp. 146-155, 2006.

[32] L. Gao, J. P. Aguiar-Moya, and Z. Zhang, "Bayesian analysis of heterogeneity in modeling of pavement fatigue cracking," Journal of Computing in Civil Engineering, vol. 26, no. 1, pp. 3743, 2012.

[33] M. Attalla, T. Hegazy, and R. Haas, "Reconstruction of the building infrastructure: two performance prediction models," Journal of Infrastructure Systems, vol. 9, no. 1, pp. 26-34, 2003.

[34] J. N. Tack, Geostatistically Based Pavement Performance Prediction Using Universal Kriging and Artificial Neural Networks [Ph.D. thesis], University of Toledo, Toledo, USA, 2002.

[35] Y.-H. Huang, "Artificial neural network model of bridge deterioration," Journal of Performance of Constructed Facilities, vol. 24, no. 6, pp. 597-602, 2010.

[36] V. Karwa and E. T. Donnell, "Predicting pavement marking retroreflectivity using artificial neural networks: Exploratory analysis," Journal of Transportation Engineering, vol. 137, no. 2, pp. 91-103, 2010.

[37] B. Saghafi, A. Hassani, R. Noori, and M. G. Bustos, "Artificial neural networks and regression analysis for predicting faulting in jointed concrete pavements considering base condition," International Journal of Pavement Research and Technology, vol. 2, no. 1, pp. 20-25, 2009.

[38] S. R. Amin and L. E. Amador-Jiménez, "Backpropagation Neural Network to estimate pavement performance: dealing with measurement errors," Road Materials and Pavement Design, pp. $1-21,2016$.

[39] H. Ziari, J. Sobhani, J. Ayoubinejad, and T. Hartmann, "Prediction of IRI in short and long terms for flexible pavements: ANN and GMDH methods," International Journal of Pavement Engineering, vol. 17, no. 9, pp. 776-788, 2015.

[40] Y. C. Tsai, Y. C. Wu, and C. Wang, "Georgia Concrete Pavement Performance and Longevity," Report No.10-10, Georgia Department of Transportation, Atlanta, USA, 2012.

[41] W.-N. Wang and Y.-C. J. Tsai, "Back-propagation network modeling for concrete pavement faulting using LTPP data," International Journal of Pavement Research and Technology, vol. 6, no. 5, pp. 651-657, 2013. 
[42] W. N. Wang, Analysis of Design Factors Impacting Joint Plain Concrete Pavement Faulting and Development of Forecasting Model Using LTPP Data [Ph.D. thesis], Changan University, Xi'an, Shaanxi, China, 2014.

[43] Y. B. Lv and H. M. Peng, "Forecasting the trend of oil production of talimu oilfield in the near future by using markov chain," Journal of Xian Shiyou University (Natural Science Edition), vol. 19, no. 4, pp. 77-79, 2004.

[44] A. Kumar and V. K. Minocha, "Rainfall runoff modeling using artificial neural networks," Journal of Hydrologic Engineering, vol. 6, no. 2, pp. 176-177, 2001.

[45] D. T. Thube, "Artificial Neural Network (ANN) based pavement deterioration models for low volume roads in India," International Journal of Pavement Research and Technology, vol. 5, no. 2, pp. 115-120, 2012.

[46] C. Chen and J. Zhang, "Comparisons of IRI-based pavement deterioration prediction models using new mexico pavement data," in Proceedings of the Geo-Frontiers 2011: Advances in Geotechnical Engineering, pp. 4594-4603, usa, March 2011. 

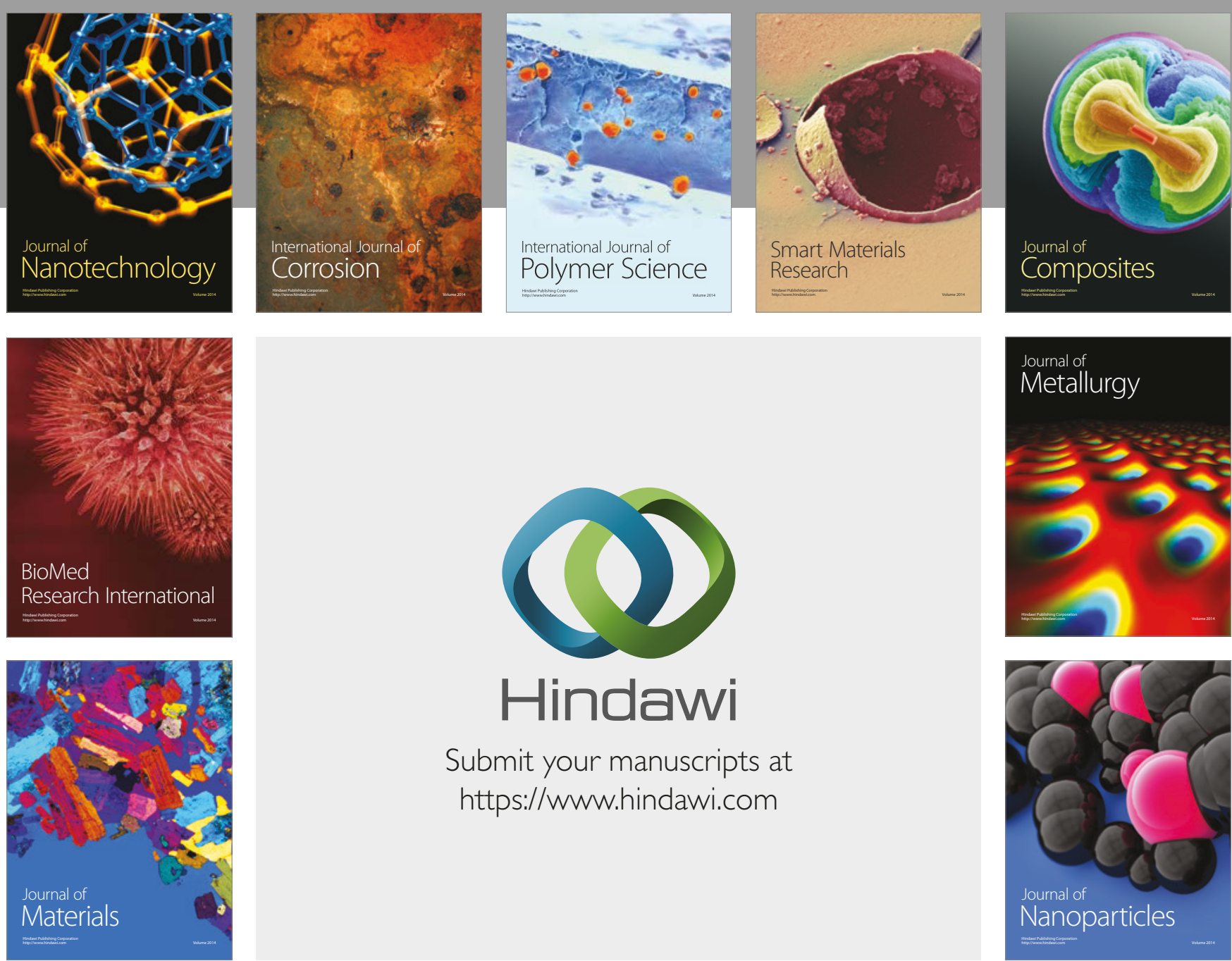

\section{Hindawi}

Submit your manuscripts at

https://www.hindawi.com
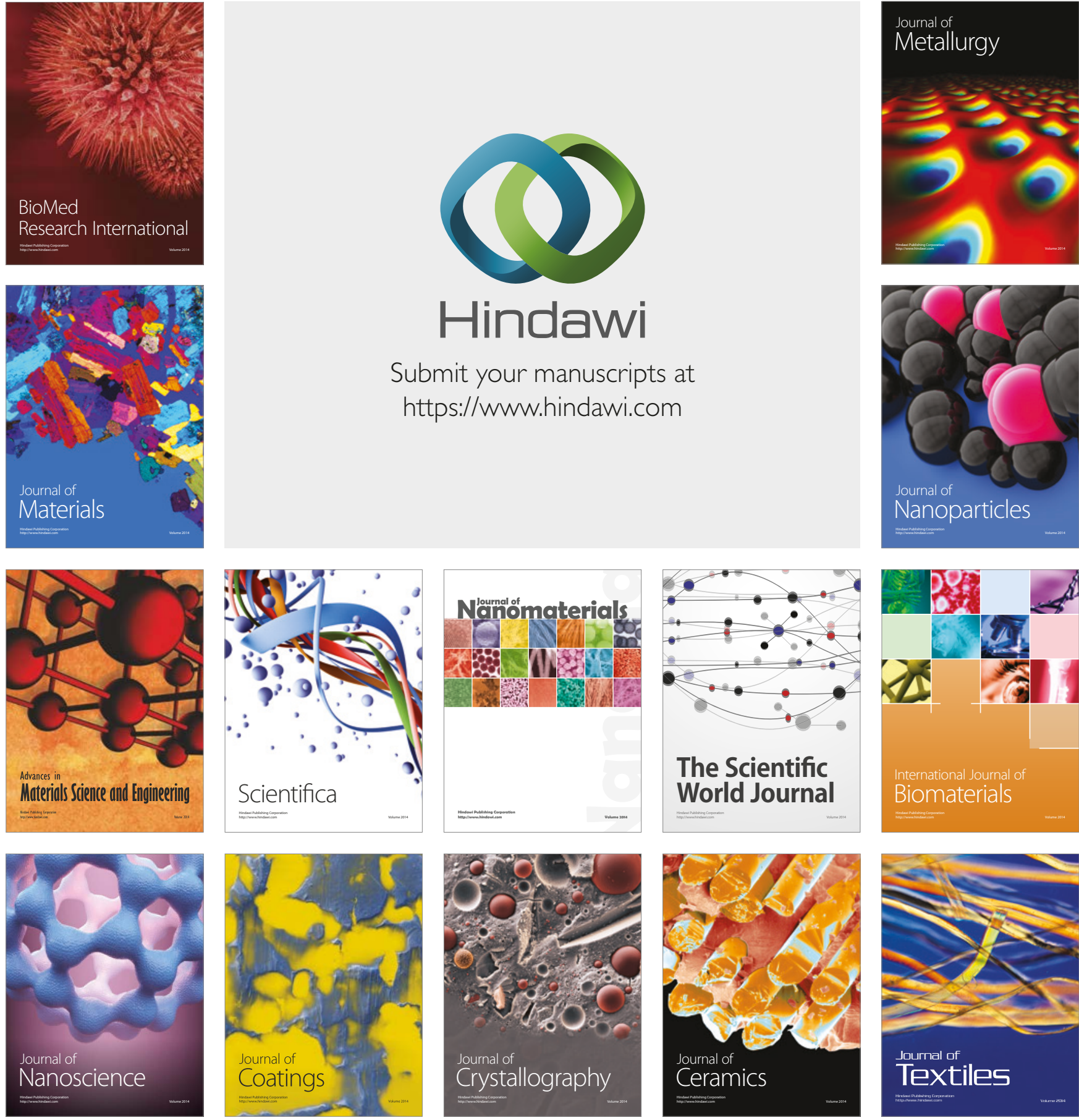

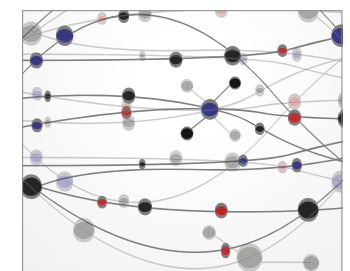

The Scientific World Journal
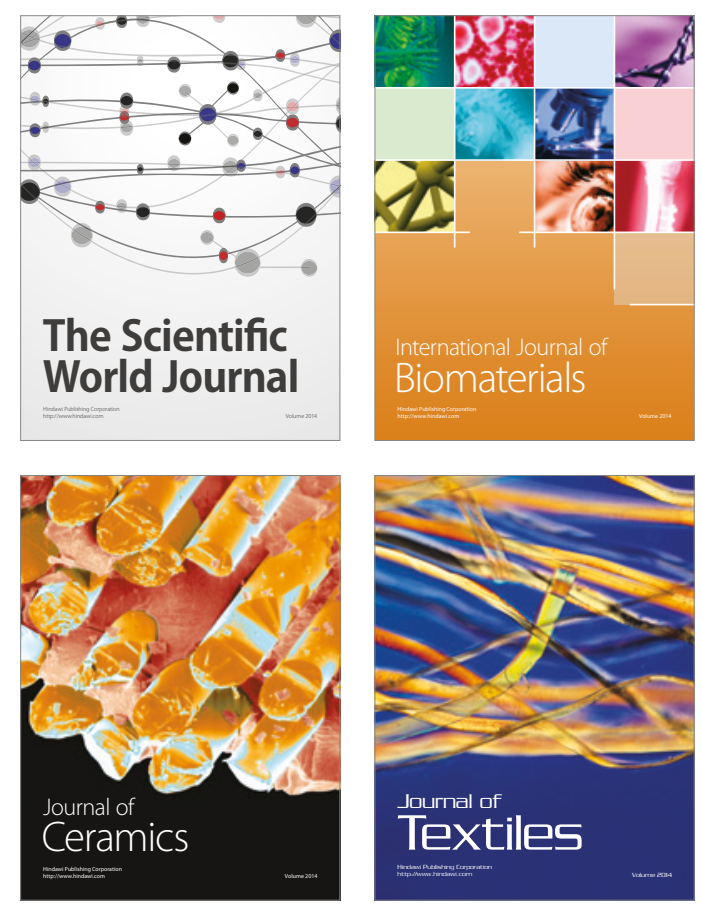